\title{
Controllable finite ultra-narrow quality-factor peak in a perturbed Dirac-cone band structure of a photonic crystal slab
}

\author{
Alex Y. Song, ${ }^{1}$ Akhil Raj Kumar Kalapala, ${ }^{2}$ Ricky Gibson, ${ }^{3}$ Kevin James \\ Reilly, ${ }^{4}$ Thomas Rotter, ${ }^{4}$ Sadhvikas Addamane, ${ }^{4}$ Haiwen Wang, ${ }^{5}$ Cheng Guo, ${ }^{5}$ \\ Ganesh Balakrishnan, ${ }^{4}$ Robert Bedford, ${ }^{3}$ Weidong Zhou, ${ }^{2}$ and Shanhui Fan ${ }^{1, *}$ \\ ${ }^{1}$ Department of Electrical Engineering, Stanford University, Stanford, California 94305, USA \\ ${ }^{2}$ Department of Electrical Engineering, University of Texas at Arlington, Arlington, Texas 76019, USA \\ ${ }^{3}$ Air Force Research Laboratory, Wright-Patterson AFB, Dayton, OH 45433, USA \\ ${ }^{4}$ Department of Electrical and Computer Engineering, \\ University of New Mexico, Albuquerque, NM 87131, USA \\ ${ }^{5}$ Department of Applied Physics, Stanford University, Stanford, California 94305, USA
}

(Dated: 21st April 2021)

\begin{abstract}
We show that by using a perturbed photonic Dirac-cone, one can realize ultra-narrow and finite $Q$-factor peak in the wavevector space, with both the peak value and the width separately tunable. We also discuss a lower bound in the minimal viable width given a peak $Q$-value while maintaining sufficient $Q$ differentiation among modes. The strong angular and frequency $Q$-selection finds applications in optical devices where strong angle- and frequency-selection is needed.
\end{abstract}

Engineering the photonic band structure has led to significant advances in the developments of optoelectronic devices [1-3]. Most band-structure engineering focuses on the eigenfrequency of the modes as a function of the wavevector. Recently on the other hand, engineering the quality-factor ( $Q$-factor) as a function of the wavevector has drawn increasing interest due to a number of applications such as light-trapping, two-dimensional lasing, and meta optics [4-12]. One of the desired features in such engineering is to obtain a fully-controllable finite and narrow high- $Q$ peak. This allows the devices to interact with the electromagnetic waves only at a certain angle and frequency, which is important in angle-selective optical devices such as absorbers [13-24] and thermal emitters [25-38]. This feature is also useful in achieving single-mode large-area lasing for high-power applications. Such devices have always been challenging since as the device-size scales, the spacing between the modes in the wavevector space reduces, leading to a diminishing $Q$ - and hence threshold-difference [39-43].

By exploring a perturbed photonic Dirac-cone [44-46], in this Letter we demonstrate a ultra-narrow finite $Q$ peak in a photonic-crystal slab. Both the peak $Q$-factor and its width in the wavevector space are independently controllable. Our approach exploits the strong mixing between the Dirac-cone bands to the linear order in the wavevector. Such mixing leads to a drastic $Q$ reduction away from the Brillouin zone center $\Gamma$. The peak $Q$ can be tuned by the strength of the perturbation, i.e. the size of the additional small holes in the photonic-crystal slab. The width of the peak can be tuned by the thickness of the slab. We also derive a trade-off relation between the peak $Q$ value and the width, which gives a lower bound of the latter given the former. Our construction can be used to fabricate perfect absorbers that are both directionand frequency-selective. The design of surface-emitting lasers can also benefit from this result, as a controllable outstanding high- $Q$ mode at $\Gamma$ promotes single-mode lasing in a scaled device.

\section{MAIN RESULT}

To motivate our results, we consider generically how a peaked $Q$ can be obtained. In the following, we examine optical resonators with light incident or output in the out-of-plane direction, with $\boldsymbol{k}=\left(k_{x}, k_{y}\right)$ representing the 2D in-plane wavevector, as is illustrated in Fig. 1. Our goal is to obtain a peaked $Q$ as a function of the angles. We start with a uniform dielectric slab, which can be found in vertical-cavity surface-emitting lasers (VCSELs). There are Fabre-Pérot resonances in the vertical direction in the slab. Their $Q$-factors as a function of the wave incident angle is nearly constant near the nor-
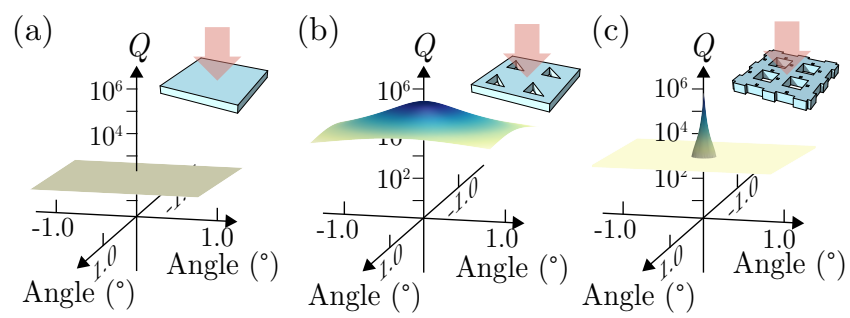

Figure 1. Comparison of the angular $Q$ variation in different structures. (a) A Fabry-Pérot band at the frequency of $0.5 \mathrm{c} / \mathrm{d}$ in a uniform slab of thickness $d$ and permittivity of 12 , where $c$ is the light speed. (b) A guided resonance mode at the frequency of $0.36 c / l$ in a photonic-crystal slab, where $l$ is the periodicity of the photonic-crystal. The slab has a thickness of $0.8 \mathrm{l}$. The side length of the isosceles right triangle holes is $0.5 l$. (c) The quadrupole band for the perturbed photoniccrystal slab structure shown in Fig. 2. 
mal direction, as is shown in Fig. 1a. This can be improved by introducing a $2 \mathrm{D}$ photonic crystal in the slab, as is illustrated in Fig. 1b. Here, the modes at non-zero angles have a lower $Q$ than the mode at the normal direction, i.e. the Brillouin zone center $\Gamma$. When used in a photonic-crystal surface-emitting laser (PCSEL), the mode at $\Gamma$ would exhibit a lower threshold than other modes. This is in accordance with the recent success of PCSELs, where single-mode lasing is maintained in a much larger area compared to the VCSELs [8, 42, 43].

Our task here is to further narrow the $Q(\boldsymbol{k})$ peak to such as the one shown in Fig. 1c. Our design concept is as follows. In a photonic-crystal slab, there exists some high- $Q$ modes and low- $Q$ modes at $\Gamma$. We can further assume that some modes have different symmetries at $\Gamma$, such that they won't mix and moreover it is possible to tune the structure so that the real part of the frequency of these modes are degenerate. At a non-zero $\boldsymbol{k}$, since the symmetry is lowered from $\Gamma$, these modes starts to mix with each other. Generically, such a mixing leads to a high- $Q$ peak at $\Gamma$. The width of the peak is controlled by the strength of the $\boldsymbol{k} \cdot \boldsymbol{p}$ terms that mix the modes [46]. At small $\boldsymbol{k}$, the strongest possible such term is to the linear order in $\boldsymbol{k}$. If we consider a high- $Q$ mode $|q\rangle$ and a low- $Q$ mode $|d\rangle$ at $\Gamma$, generically, the narrowest $Q$ peak should be described by the following effective $\boldsymbol{k} \cdot \boldsymbol{p}$ Hamiltonian

$$
h(k)=\left(\begin{array}{cc}
i \gamma_{d} & i v_{g} k \\
-i v_{g} k & i \gamma_{q}
\end{array}\right),
$$

along a direction $\boldsymbol{k}$ in the Brillouin zone. Here $k=|\boldsymbol{k}|$ is the magnitude of the in-plane wavevector, $v_{g}$ is the group velocity of the bands, $\gamma_{d}$ is the radiation constant of the low- $Q$ mode at $\Gamma$, and $\gamma_{q}$ is that of the high- $Q$ mode at $\Gamma$. The resulting $Q(\boldsymbol{k})$ function for the band containing mode $|q\rangle$ is plotted in Fig. 1c, assuming the parameters of $\gamma_{q}=6.7 \times 10^{-7}, \gamma_{d}=3.8 \times 10^{-4}$, and $v_{g}=0.11 \mathrm{c} / 2 \pi$. Due to the strong mixing that is linear in $\boldsymbol{k}$, the $Q$ factor reduces drastically away from $\Gamma$, leading to a finite and narrow $Q$ peak.

The remaining task is to design a structure that gives the effective Hamiltonian in Eq. (1). The linear mixing in Eq. (1) corresponds to a Dirac-cone band structure $[44,45]$. In a photonic crystal slab, one can form a Dirac cone by creating an accidental degeneracy at the $\Gamma$ point between a pair of two-fold degenerate dipole states and a singly degenerate state $[44,45,47]$. However, in all previous works, due to the symmetry of the structure used, the singly degenerate state has an infinite $Q$. The resulting system thus does not have the desired $Q(\boldsymbol{k})$ dependency [46]. Our approach is to start with a Dirac-cone formed by the approach described above and perturb it such that the infinite- $Q$ mode becomes radiative.

To implement our approach, we consider the photoniccrystal slab structure as is shown in the inset of Fig. 2a. The photonic-crystal slab has a square lattice with period
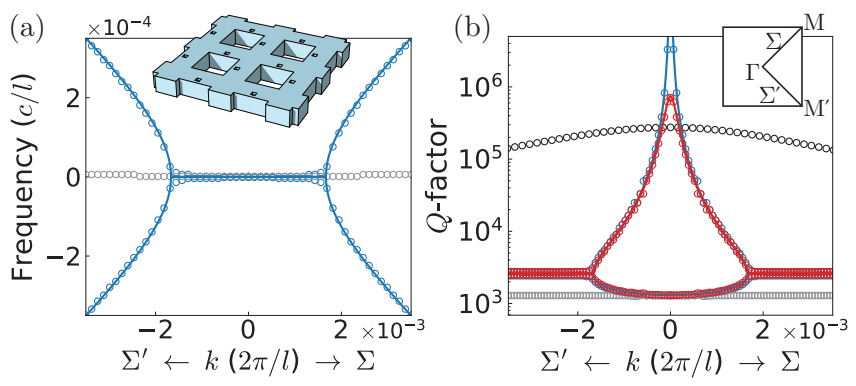

Figure 2. Band structure and the $Q(\boldsymbol{k})$ function of the photonic Dirac-cone. Circles represent numerical calculation results, while solid curves represent results of the effective model in Eq. (1). (a) band structure of the unperturbed structure. Two dispersive (blue) bands and the flat-band (gray) are plotted. Inset: schematic of the photonic-crystal slab. The frequency is offset to the Dirac-cone frequency at $0.496 c / l$. (b) $Q(\boldsymbol{k})$ functions of the unperturbed structure (blue) and the perturbed (red). The flat-band is plotted in Grey circles. Black circles represent the $Q(\boldsymbol{k})$ function of the photonic-crystal slab with triangular holes. Inset: diagram of the first Brillouin zone.

$l$ in both $x$ and $y$ directions. We normalize all the other geometrical parameters in units of $l$. The slab has a thickness of $t=0.8 l$, and a permittivity of 12 as is typical for common semiconductors in the infrared wavelength range. In each unit-cell, there is a large square-shaped hole at the center with a side length of $a_{1}$ and two smaller holes with a side length of $a_{2}=0.05 l$ located at $(0.4 l, 0)$ and $(0,0.4 l)$, respectively. The large hole is through the slab, while the smaller holes have a depth of $t_{2}=0.05 l$. Without the smaller holes, the PhC slab has a $D_{4 h}$ pointgroup symmetry [48]. The symmetry reduces to $C_{2 v}$ with the smaller holes added.

The band structure and the $Q(\boldsymbol{k})$ functions can be directly calculated by monitoring the poles of the scattering matrix on the complex frequency plane [49] [50]. The results are plotted as blue circles in Fig. $2 \mathrm{a}$ and b, respectively. In the absence of the smaller holes, and with a side length of $a_{1}=0.69 \mathrm{l}$, three modes are tuned to degeneracy at $\Gamma$. In the resulting band structure in Fig. 2, two of the bands are dispersive, while the third forms a flat band. Both the band structure in Fig. 2a and the $Q(\boldsymbol{k})$ functions in Fig. 2b show two phases separated by exceptional points [45]. At $\Gamma$, the infinite- $Q$ mode belongs to the $B_{1 g}$ representation of the $D_{4 h}$ point group and is of quadrupole nature. The low- $Q$ mode belong to the $E_{u}$ representation, which is of dipolar nature and strongly couples to free-space radiation. The resulting $Q(\boldsymbol{k})$ is sharply peaked for one of the bands. However, the mode at $\Gamma$ in this band has a infinite $Q$-factor and is a bound state in the continuum (BIC) [5], which can not couple to free space to perform light detection, absorption, or emission.

To create a sharply peaked $Q(\boldsymbol{k})$ where $Q$ is finite at the peak, we perturb the structure by introducing two 
smaller holes as mentioned above to break the $D_{4 h}$ symmetry, such that the quadrupole mode is allowed to radiate. With the perturbation, the real part of the band structure is virtually identical to the the one without in Fig. 2a, hence is not plotted. The $Q(\boldsymbol{k})$ functions with the perturbation are shown as red circles in Fig. 2b. We observe that the $Q(\boldsymbol{k})$ functions for both the high- $Q$ and the low- $Q$ bands are very close to those without perturbation, except near the Brillouin zone center $\Gamma$. At $\Gamma$, the high- $Q$ mode now has a finite $Q$-factor rather than infinite. Away from $\Gamma$, the $Q$-factor quickly reduces, thus forming a sharp peak at $\Gamma$. As a reference, the $Q(\boldsymbol{k})$ function of the band at the frequency of $0.36 \mathrm{c} / \mathrm{l}$ in a photonic-crystal slab with a square lattice and triangular holes is also plotted in the black curve in Fig. 2b [8, 49]. This is a singly degenerate band with no other bands nearby in frequency, hence does not form a Dirac-cone. A schematic of this structure can be found in Fig. 1 b. The peak $Q$-factor is at a level of $10^{5}$, similar to our design here. However, The $Q(\boldsymbol{k})$ function is much more slowly varying. The $Q$ peak width is reduced by an order of magnitude in our work through exploiting the strong linear-order mixing.

The band structure and the $Q(\boldsymbol{k})$ function of the two dispersive bands are described by the effective Hamiltonian in Eq. (1) [45]. Here, $\gamma_{d}$ and $\gamma_{q}$ can be read out from the numerical calculations at the Brillouin zone center $\Gamma$. $v_{g}$ can be obtained by fitting to the slope of the bands outside of the exceptional points. The complex eigenfrequencies of the Dirac-cone bands are then obtained by solving Eq. (1). The resulting band structure and the $Q(\boldsymbol{k})$ functions are plotted as solid curves in Fig. 2. The results very accurately reproduce the numerical simulations. In existing studies of the photonic Dirac-cones, $\gamma_{q}$ is zero, leading to an infinite $Q$-factor. In our work, $\gamma_{q}$ becomes finite due to the introduction of the smaller holes. The perturbation can also cause small corrections to other parameters in Eq. 1, which are omitted in the lowest order. From Eq. (1) it is clear that the offdiagonal terms that is linear in $\boldsymbol{k}$ causes a strong mixing between the dipolar and the quadrupole modes, resulting in a sharp $Q$ peak. It is important to note that the entire band structure and the $Q(\boldsymbol{k})$ functions are controlled by only three parameters, i.e. $\gamma_{d}, \gamma_{q}$, and $v_{g}$.

\section{INDEPENDENT ENGINEERING OF THE PEAK $Q$ AND ITS WIDTH}

In our approach to engineer the $Q(\boldsymbol{k})$ function, both the peak $Q$ and its width can be tuned. The peak $Q$ value can be straightforwardly engineered by controlling the size of the small holes. For example, In Fig. 3(a) we plot the $Q(\boldsymbol{k})$ function of the same structure as above, but with the small holes having a reduced side length and depth of $a_{2}=t_{2}=0.04 l$. The resulting $Q$-factor at $\Gamma$
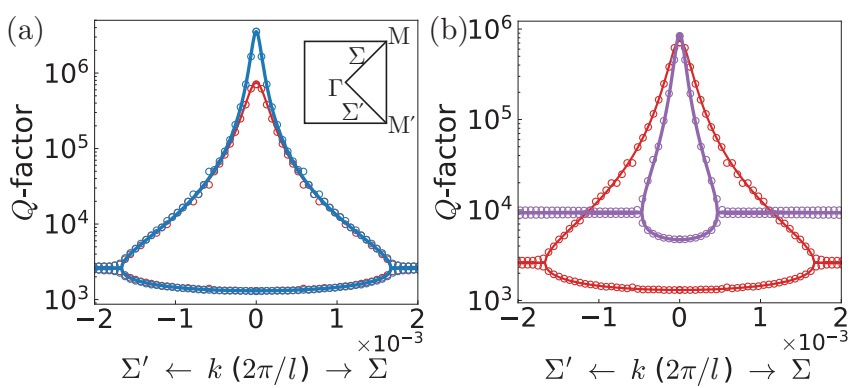

Figure 3. (a) The $Q(\boldsymbol{k})$ functions of the photonic-crystal slab with the side length and depth of the small holes to be $0.05 l$ (red) and $0.04 l$ (blue), respectively. (b) The $Q(\boldsymbol{k})$ functions of the photonic-crystal slab with a thickness of $0.8 l$ (red) and $0.775 l$ (purple), respectively. The circles are results of the numerical calculation. The solid curves are results of the effective model in Eq. (1).

now increases by an order of magnitude while the width of the peak remains largely unchanged.

To tune the width of the $Q$ peak without changing the peak value, we can change the $Q$-factor of the dipolar mode at $\Gamma$. As is discussed above, the $Q(\boldsymbol{k})$ functions of both bands are controlled by only three parameters, i.e. $\gamma_{q}, \gamma_{d}$, and $v_{g}$. By increasing the $Q$-factor of the dipolar mode, the region inside the exceptional points shrinks, leading to a reduced peak width. A demonstration is shown in Fig. 3(b), in which we compare the $Q(\boldsymbol{k})$ function of the design above that corresponds to the red curve in Fig. 3a and one with a reduced slab thickness of $t=0.775 l$ (purple). The side length of the large hole is adjusted to $0.695 l$ to maintain the degeneracy of the real part of the bands. We also tune the smaller holes to have a side length and depth of $a_{2}=t_{2}=0.035 l$, to maintain the peak $Q$-value at $\Gamma$. The resulting $Q(\boldsymbol{k})$ functions of both numerical calculation (circles) and the analytical model (solid curves) are shown. The $Q$-factor of the dipolar mode increased by four times in the thinner photonic-crystal slab. As a result, the high- $Q$ bandwidth reduces by a factor of 2 while the peak $Q$ value is unchanged, as is observed in Fig. 3(b).

\section{PEAK-WIDTH RELATION}

The above discussion of the tuning of the high- $Q$ width gives a hint of a relation between the minimal viable width and the peak $Q$. In real devices, it is often desirable to design a certain $Q$-factor at the zone center $\Gamma$ and at the same time a strong $Q$-differentiation against other modes away from $\Gamma$. For simplicity, We define the width of the high- $Q$ region by the width of the wavevector region inside the exceptional points. The location of the exceptional point can be calculated from Eq. (1) as $k_{e}=\left(\gamma_{d}-\gamma_{q}\right) / 2 v_{g}$. It is clear that a higher $Q_{d}$, i.e. a smaller $\gamma_{d}$, leads to a smaller $k_{e}$. By the requirement of 


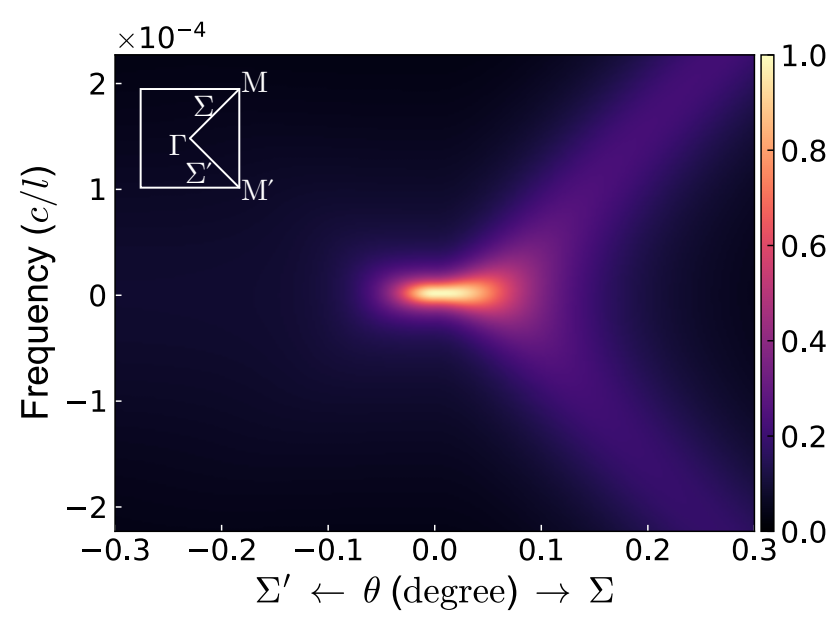

Figure 4. The absorption spectra of the angle- and frequency-selective absorber. The frequency is offset to the Dirac-cone point at $0.485(c / l)$. The incident wave is polarized such that the in-plane projection of the electric field is pointed in the $45^{\circ}$ direction.

$Q$-differentiation, we consider that the peak $Q$ value at $\Gamma$ is at least twice the value outside the exceptional points. The minimal $\gamma_{d}$ is then given by $\gamma_{d}=3 \gamma_{q}$. This leads to a minimal width as

$$
w_{\min }=2 k_{e}=2 \frac{\omega}{Q_{\max } \cdot v_{g}}
$$

where $\omega$ is the frequency of the Dirac point, $Q_{\max }$ is the desired peak quality factor at $\Gamma$, and $v_{g}$ is the group velocity of the Dirac-cone bands. For a target $Q$-factor at $\Gamma$, Eq. (2) thus gives the lower bound on the minimal peak width while maintaining sufficient $Q$ differential from the $\Gamma$ point to other wave vectors.

\section{ANGLE- AND FREQUENCY-SELECTIVE PERFECT ABSORPTION}

A sharply peaked $Q(\boldsymbol{k})$ function is useful in a variety of scenarios where strong angle- and frequencydifferentiation is needed, such as angle-selective sensors or large-area single-mode lasers. As an example, here we demonstrate a perfect absorber based on the photoniccrystal slab with a strong selection in both the frequency and the incident angle. The geometric parameters of the slab is as follows. The slab has a thickness of $t=0.85 l$, the large hole has a side length of $0.682 l$, and the small holes have a depth and a side length of $0.1 \mathrm{l}$. We add a mirror to the bottom of the photonic-crystal slab at a distance of $20.3 \mathrm{l}$. Light incidence is from the top. We make use of the quadrupole mode at $\Gamma$, which is an outstanding high- $Q$ point in a parameter space of three dimensions, i.e. frequency plus $2 \mathrm{D}$ solid angles. We assume a uniform material loss in the photonic-crystal slab by adding a constant imaginary part to its refractive index, i.e. $\varepsilon=12-4.8 \times 10^{-5} i$, representing a semiconductor interband absorption which is typically much broader than the optical resonance. Such a material absorption provides a modal internal loss to the quadrupole mode at $\Gamma$ in the same amount as the radiative loss, and hence resulting in a perfect absorption due to critical coupling $[51,52]$. At any other angle or frequency, such condition is not met and the absorption is low. The numerical results are shown in Fig. 4, where we incident light on to the structure with varying frequencies and angles. Here, light is assumed to be polarized such that the in-plane projection of the electric field is pointed in the $45^{\circ}$ direction, i.e. it is $p$-polarized for the spectra along $\Sigma$ and $s$-polarized along $\Sigma^{\prime}$. It is observed that strong absorption only occurs within an angle of $0.1^{\circ}$ from normal, in a narrow frequency range of $10^{-5} c / l$. Again, the width of the angle can be tuned by changing the $Q$-factor of the dipolar mode, for example by tuning the slab thickness. We note that the adding of the bottom mirror can introduce a correction to the frequency and the radiative constant of both the dipolar and the quadrupole bands [53]. Yet, this would not affect the narrowing of the $Q$ peak in the perturbed Dirac-cone.

\section{CONCLUSION}

In summary, in this Letter we presented an approach to obtain a finte and ultra-narrow $Q$ peak, with the peak value and width independently tunable. This is realized by employing a perturbed photonic Dirac-cone. Utilizing the linear mixing between modes in the Dirac-cone, the modes at $\Gamma$ strongly remix away from $\Gamma$, leading to a rapidly decreasing $Q(\boldsymbol{k})$ function for a high- $Q$ band. The narrow $Q$ peak is useful in scenarios where angular selection is needed, such as directional absorber or emitter. The feature can be useful in surface-emitting lasers as well, since a large $Q$ differentiation promotes single-mode lasing. Although we focus on $2 \mathrm{D} \mathrm{PhC}$ structure, this approach applies to other dimensions such as 1D distributed Bragg reflectors (DBRs).

This work is supported in part by the Department of Defense Joint Directed Energy Transition Office (DEJTO) under Grant No. N00014-17-1-2557.

*shanhui@stanford.edu

[1] Kazuaki Sakoda, Optical properties of photonic crystals, Vol. 80 (Springer Science \& Business Media, 2004).

[2] John D. Joannopoulos, Steven G. Johnson, Joshua N. Winn, and Robert D. Meade, Photonic Crystals: Molding the Flow of Light, 2nd ed. (Princeton University Press, USA, 2008). 
[3] William L Barnes, Alain Dereux, and Thomas W Ebbesen, "Surface plasmon subwavelength optics," nature 424, 824-830 (2003).

[4] Jicheng Jin, Xuefan Yin, Liangfu Ni, Marin Soljačić, Bo Zhen, and Chao Peng, "Topologically enabled ultrahigh-q guided resonances robust to out-of-plane scattering," Nature 574, 501-504 (2019).

[5] Chia Wei Hsu, Bo Zhen, A Douglas Stone, John D Joannopoulos, and Marin Soljačić, "Bound states in the continuum," Nature Reviews Materials 1, 1-13 (2016).

[6] Ashok Kodigala, Thomas Lepetit, Qing Gu, Babak Bahari, Yeshaiahu Fainman, and Boubacar Kanté, "Lasing action from photonic bound states in continuum," Nature 541, 196-199 (2017).

[7] Mario I. Molina, Andrey E. Miroshnichenko, and Yuri S. Kivshar, "Surface bound states in the continuum," Phys. Rev. Lett. 108, 070401 (2012).

[8] Kazuyoshi Hirose, Yong Liang, Yoshitaka Kurosaka, Akiyoshi Watanabe, Takahiro Sugiyama, and Susumu Noda, "Watt-class high-power, high-beam-quality photonic-crystal lasers," Nature photonics 8, 406-411 (2014).

[9] Kirill Koshelev, Sergey Lepeshov, Mingkai Liu, Andrey Bogdanov, and Yuri Kivshar, "Asymmetric metasurfaces with high-q resonances governed by bound states in the continuum," Physical review letters 121, 193903 (2018).

[10] Peter Bermel, Chiyan Luo, Lirong Zeng, Lionel C. Kimerling, and John D. Joannopoulos, "Improving thin-film crystalline silicon solar cell efficiencies with photonic crystals," Opt. Express 15, 16986-17000 (2007).

[11] Ranjan Singh, Wei Cao, Ibraheem Al-Naib, Longqing Cong, Withawat Withayachumnankul, and Weili Zhang, "Ultrasensitive terahertz sensing with high-q fano resonances in metasurfaces," Applied Physics Letters 105, 171101 (2014).

[12] Son Tung Ha, Yuan Hsing Fu, Naresh Kumar Emani, Zhenying Pan, Reuben M Bakker, Ramón PaniaguaDomínguez, and Arseniy I Kuznetsov, "Directional lasing in resonant semiconductor nanoantenna arrays," Nature nanotechnology 13, 1042-1047 (2018).

[13] Yasuhiko Takeda, Taichi Maruya, and Hideyuki Tanaka, "Absorptive angular-selective filters consisting of dielectric multilayers combined with thin absorbing layers," Applied Optics 58, 9094 (2019).

[14] Linxiao Zhu, Fengyuan Liu, Hongtao Lin, Juejun Hu, Zongfu Yu, Xinran Wang, and Shanhui Fan, "Angleselective perfect absorption with two-dimensional materials," Light: Science \& Applications 5, e16052-e16052 (2016).

[15] Zhiguang Zhou, Enas Sakr, Yubo Sun, and Peter Bermel, "Solar thermophotovoltaics: Reshaping the solar spectrum," Nanophotonics 5, 1-21 (2016).

[16] Emily D. Kosten, Jackson H Atwater, James Parsons, Albert Polman, and Harry A. Atwater, "Highly efficient GaAs solar cells by limiting light emission angle," Light: Science \& Applications 2, e45-e45 (2013).

[17] Chan-Sol Hwang, Sung-Pyo Yang, Kyung-Won Jang, Jung-Woo Park, and Ki-Hun Jeong, "Angle-selective optical filter for highly sensitive reflection photoplethysmogram," Biomedical Optics Express 8, 4361 (2017).

[18] Subaru Morita, Hidehisa Shiomi, Hiroshi Murata, and Atsushi Sanada, "Highly angle and frequency selective absorption by mushroom metasurfaces for indoor propagation control," 2017 IEEE MTT-S International
Conference on Microwaves for Intelligent Mobility, ICMIM 2017 , 68-70 (2017).

[19] Yasuhiko Takeda, Hideo Iizuka, Noboru Yamada, and Tadashi Ito, "Light trapping for photovoltaic cells using polarization-insensitive angle-selective filters under monochromatic illumination," Applied Optics 56, 5761 (2017).

[20] Byung Hoon Woo, In Cheol Seo, Eunsongyi Lee, SooChan An, Hoon Yeub Jeong, and Young Chul Jun, "Angle-dependent optical perfect absorption and enhanced photoluminescence in excitonic thin films," Optics Express 25, 28619 (2017).

[21] Y. Shen, D. Ye, I. Celanovic, S. G. Johnson, J. D. Joannopoulos, and M. Solja i, "Optical Broadband Angular Selectivity," Science 343, 1499-1501 (2014).

[22] Enas Sakr and Peter Bermel, "Angle-Selective Reflective Filters for Exclusion of Background Thermal Emission," Physical Review Applied 7, 044020 (2017).

[23] Rafif E. Hamam, Ivan Celanovic, and Marin Soljačić, "Angular photonic band gap," Physical Review A 83, 035806 (2011).

[24] Jessica R. Piper and Shanhui Fan, "Total absorption in a graphene monolayer in the optical regime by critical coupling with a photonic crystal guided resonance," ACS Photonics 1, 347-353 (2014).

[25] Denis G Baranov, Yuzhe Xiao, Igor A Nechepurenko, Alex Krasnok, Andrea Alù, and Mikhail A Kats, "Nanophotonic engineering of far-field thermal emitters," Nature materials , 1 (2019).

[26] D. Costantini, A. Lefebvre, A.-L. Coutrot, I. MoldovanDoyen, J.-P. Hugonin, S. Boutami, F. Marquier, H. Benisty, and J.-J. Greffet, "Plasmonic metasurface for directional and frequency-selective thermal emission," Phys. Rev. Applied 4, 014023 (2015).

[27] J. A. Mason, S. Smith, and D. Wasserman, "Strong absorption and selective thermal emission from a midinfrared metamaterial," Applied Physics Letters 98, 241105 (2011).

[28] Enas Sakr and Peter Bermel, "Thermophotovoltaics with spectral and angular selective doped-oxide thermal emitters," Opt. Express 25, A880-A895 (2017).

[29] Moisés Garín, David Hernández, Trifon Trifonov, and Ramón Alcubilla, "Three-dimensional metallo-dielectric selective thermal emitters with high-temperature stability for thermophotovoltaic applications," Solar Energy Materials and Solar Cells 134, 22 - 28 (2015).

[30] Xingxing Liu, Zhiwei Li, Zhengji Wen, Mingfei Wu, Jialiang Lu, Xu Chen, Xinchao Zhao, Tao Wang, Ruonan Ji, Yafeng Zhang, Liaoxin Sun, Bo Zhang, Hao Xu, Jing Zhou, Jiaming Hao, Shaowei Wang, Xiaoshuang Chen, Ning Dai, Wei Lu, and Xuechu Shen, "Largearea, lithography-free, narrow-band and highly directional thermal emitter," Nanoscale 11, 19742-19750 (2019).

[31] Salvatore Campione, Francois Marquier, Jean-Paul Hugonin, A Robert Ellis, John F Klem, Michael B Sinclair, and Ting S Luk, "Directional and monochromatic thermal emitter from epsilon-near-zero conditions in semiconductor hyperbolic metamaterials," Scientific reports 6, 1-9 (2016).

[32] Jean-Jacques Greffet, Rémi Carminati, Karl Joulain, Jean-Philippe Mulet, Stéphane Mainguy, and Yong Chen, "Coherent emission of light by thermal sources," Nature 416, 61-64 (2002). 
[33] S. E. Han and D. J. Norris, "Beaming thermal emission from hot metallic bull's eyes," Opt. Express 18, 48294837 (2010).

[34] Christophe Arnold, Fran çois Marquier, Moisés Garin, Fabrice Pardo, Stéphane Collin, Nathalie Bardou, JeanLuc Pelouard, and Jean-Jacques Greffet, "Coherent thermal infrared emission by two-dimensional silicon carbide gratings," Phys. Rev. B 86, 035316 (2012).

[35] Menaka De Zoysa, Takashi Asano, Keita Mochizuki, Ardavan Oskooi, Takuya Inoue, and Susumu Noda, "Conversion of broadband to narrowband thermal emission through energy recycling," Nature Photonics 6, 535-539 (2012).

[36] Ivan Celanovic, David Perreault, and John Kassakian, "Resonant-cavity enhanced thermal emission," Phys. Rev. B 72, 075127 (2005).

[37] Ivan Celanovic, Natalija Jovanovic, and John Kassakian, "Two-dimensional tungsten photonic crystals as selective thermal emitters," Applied Physics Letters 92, 193101 (2008).

[38] Emily D Kosten, Jackson H Atwater, James Parsons, Albert Polman, and Harry A Atwater, "Highly efficient gaas solar cells by limiting light emission angle," Light: Science \& Applications 2, e45 (2013).

[39] Shun Lien Chuang, Physics of Photonic Devices, 2nd ed. (John Wiley \& Sons, Inc., 2009) pp. 1-821.

[40] Susumu Noda, Kyoko Kitamura, Tsuyoshi Okino, Daiki Yasuda, and Yoshinori Tanaka, "Photonic-Crystal Surface-Emitting Lasers: Review and Introduction of Modulated-Photonic Crystals," IEEE Journal of Selected Topics in Quantum Electronics 23, 1-7 (2017).

[41] W. W. Chow, K. D. Choquette, M. H. Crawford, K. L. Lear, and G. R. Hadley, "Design, fabrication, and performance of infrared and visible vertical-cavity surfaceemitting lasers," IEEE Journal of Quantum Electronics 33, 1810-1824 (1997).

[42] Masahiro Yoshida, Menaka De Zoysa, Kenji Ishizaki, Yoshinori Tanaka, Masato Kawasaki, Ranko Hatsuda, Bongshik Song, John Gelleta, and Susumu Noda, "Double-lattice photonic-crystal resonators en- abling high-brightness semiconductor lasers with symmetric narrow-divergence beams," Nature Materials 18, 121-128 (2019).

[43] Takuya Inoue, Ryohei Morita, Masahiro Yoshida, Menaka De Zoysa, Yoshinori Tanaka, and Susumu Noda, "Comprehensive analysis of photonic-crystal surfaceemitting lasers via time-dependent three-dimensional coupled-wave theory," Physical Review B 99, 1-8 (2019).

[44] Xueqin Huang, Yun Lai, Zhi Hong Hang, Huihuo Zheng, and CT Chan, "Dirac cones induced by accidental degeneracy in photonic crystals and zero-refractive-index materials," Nature materials 10, 582-586 (2011).

[45] Bo Zhen, Chia Wei Hsu, Yuichi Igarashi, Ling Lu, Ido Kaminer, Adi Pick, Song-Liang Chua, John D Joannopoulos, and Marin Soljačić, "Spawning rings of exceptional points out of dirac cones," Nature 525, 354-358 (2015).

[46] Kazuaki Sakoda, "Proof of the universality of mode symmetries in creating photonic dirac cones," Optics express 20, 25181-25194 (2012).

[47] Momchil Minkov, Ian A. D. Williamson, Meng Xiao, and Shanhui Fan, "Zero-index bound states in the continuum," Phys. Rev. Lett. 121, 263901 (2018).

[48] Teturo Inui, Yukito Tanabe, and Yositaka Onodera, Group theory and its applications in physics, Vol. 78 (Springer Science \& Business Media, 2012).

[49] Alex Y Song, Akhil Raj Kumar Kalapala, Weidong Zhou, and Shanhui Fan, "First-principles simulation of photonic crystal surface-emitting lasers using rigorous coupled wave analysis," Applied Physics Letters 113, 041106 (2018).

[50] See supplemental material for more information.

[51] Herman A Haus, Waves and Fields in Optoelectronics (Prentice Hall, 1984) p. 402.

[52] Wonjoo Suh, Zheng Wang, and Shanhui Fan, "Temporal coupled-mode theory and the presence of non-orthogonal modes in lossless multimode cavities," IEEE Journal of Quantum Electronics 40, 1511-1518 (2004).

[53] Yong Xu, Yi Li, Reginald K. Lee, and Amnon Yariv, "Scattering-theory analysis of waveguide-resonator coupling," Physical Review E 62, 7389-7404 (2000). 